\title{
Line-by-line fiber Bragg grating made by femtosecond laser
}

\author{
Kaiming Zhou, Mykhaylo Dubov, Chengbo Mou, Lin Zhang, Vladimir Mezentesv, Ian Bennion
}

\begin{abstract}
In this paper, we report on the inscription of a 4th order fiber Bragg grating (FBG) made line-by-line (LBL) in the optical fiber using femtosecond (fs) laser. Strong Bragg resonance $(\sim 17 \mathrm{~dB})$ and low insertion loss $(\sim 0.5 \mathrm{~dB})$ were obtained with only 2000 periods. Measured refractive index change of these inscribed lines reaches up to $7 \times 10^{-3}$. The grating was fully characterized and the low insertion loss together with low polarization dependent loss were realized compared to the gratings made by the point-by-point (PBP) method. High temperature annealing experiment shows the grating can survive up to at least $800^{\circ} \mathrm{C}$.
\end{abstract}

Index Terms - femtosecond laser, fiber Bragg grating,

\section{INTRODUCTION}

$\mathbf{T}$ HE discovery of refractive modification of transparent material using intense femtosecond laser has led to a new technique in making photonic devices. One of the advantages of this technique is that the modification is largely independent on the properties of the material, requiring only a certain transparency to the laser, and thus the modification can be made deep within the material. Researchers have implemented a range of novel photonic devices by fs laser inscription, including optical waveguide [1], microfluidic channels [2][3] and FBGs [4], [5], [6], etc. FBGs have been made using PBP method [5], [6] or with the aid of a phase mask [4]. FBGs written with the phase mask method are mostly 2nd order gratings due to the limitation by the wavelength of the laser, which is usually Ti:sapphire laser with working wavelength around $800 \mathrm{~nm}$. The wavelength of the Bragg resonance of the FBG is also relatively inflexible since it is decided by the period of the phase mask. On the other hand, by means of focusing the fs laser pulses directly into the fiber core with an objective lens, PBP Bragg grating of arbitrary wavelegnth can be achieved without the need of a phase mask. Utilizing the nonlinear interaction of the femtosecond laser with the material, modification can be made beyond the diffraction limit and the structure size can be made smaller than the wavelength of the writing laser by controlling the pulse energy.

Previously, we have reported 1st order PBP inscribed FBGs [5], [6], the period of which is simply the ratio of the translation speed of the fiber to pulse repetition rate of the laser. Bragg wavelengths can hence be fully controlled to provide resonances within the desired range. However, these PBP inscribed FBGs show significant polarization dependent response and relatively high insertion loss. Though some

Kaiming Zhou, Chengbo Mou,Mykhaylo Dubov, Vladimir Mezentsev, Lin Zhang and Ian Bennion are with Photonic Research Group, Aston University, United Kingdom, Kaiming Zhou's email: k.zhou@aston.ac.uk. applications may desire such a polarization dependency, others nevertheless prefer non-polarization response.

In our 1st order PBP FBGs, the modified area in each period can be regarded as an ellipsoid, with a length of $2 \sim 3 \mu \mathrm{m}$ and a diameter of $\sim 500 \mathrm{~nm}$. Such a smaller feature can cause a significant scattering of the light propagating in the fiber core, which in turn induces the big loss. In reference [6], the reported loss is around $1 \mathrm{~dB}$ for a $5 \mathrm{~mm}$ long grating. The modification in each period is inscribed by only a single shot of the laser and the uniformity is unreliable because the laser energy could vary from one pulse to another. As a result, the uneven index change is produced, which implies a great level of spectral noises. In addition, due to the small size of the focused laser beam, a great degree of efforts have to be spent in the alignment to write the 1st order PBP FBGs in the center of the fiber core. This process is time consuming and demanding, as the fiber has to be aligned parallel to the direction of the motion of the translation stage and the longer grating requires more precise alignment. To write a $5 \mathrm{~mm}$ long FBG, the error has to be controlled within $1 \mathrm{mrad}$, and less than $0.2 \mathrm{mrad}$ is not uncommon for a high quality grating. In this paper, we describe LBL FBG structures inscribed by a fs laser which allows relatively coarse alignment and offers lower insertion loss and polarization dependency. Moreover, since areas of the glass modified by fs laser show significantly increased etch rate [7], lines of the LBL inscribed gratings could be etched off to produce voids and thus provide the potential for implementing novel bio/chemical sensors.

\section{EXPERIMENT AND RESULTS}

The inscription setup is the same as we used in our previous work [6], where a glass slide and index matching oil are used to eliminate the distortion to the focus of the laser by the cylindrical cladding of the optical fiber. In the experiment, fs laser pulses $(\lambda=800 \mathrm{~nm})$ are tightly focused on the fiber by a $\times 100$ objective lens with a N.A. of 0.55 and a working distance of $13 \mathrm{~mm}$. The pulse duration of the laser is $\sim 110 \mathrm{fs}$ with a $1 \mathrm{kHz}$ repetition rate. The fiber is mounted on a dualaxis air-bearing translation stage so that the desired pattern could be written by translating the fiber with respect to the focal spot of the fs-laser beam. Figure 1 is the schematic of the inscription of the LBL FBG. For each line, the focus of the laser is translated perpendicular to the axis of the fiber and through the core at a low velocity, $v_{1}$. Then the focus is moved back to the original position in the $x$ direction while in the $z$ direction it is transferred a small distance which corresponds to the period of the grating. During this 


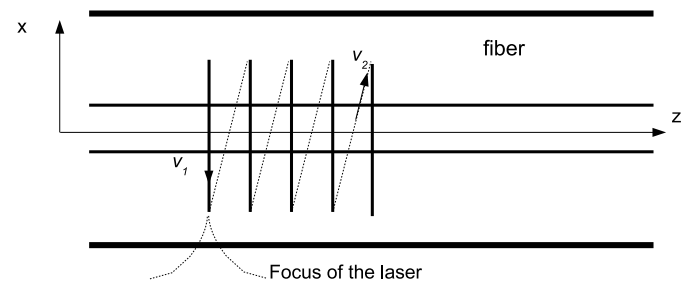

(a)

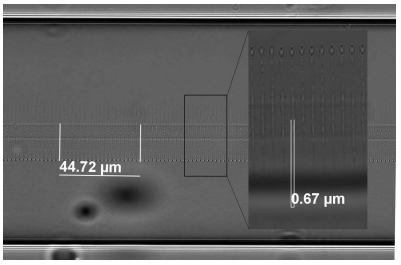

(b)

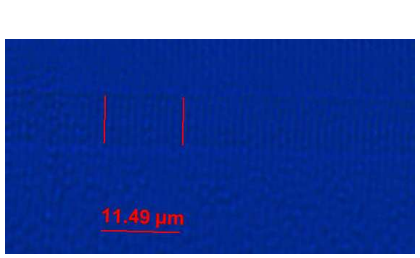

(c)
Figure 1. (a) Schematic of the inscription of the LBL FBG. (b) Image of the 4 th order LBL FBG. (c) Image of the 2nd order LBL FBG.

movement, the velocity $v_{2}$ is high enough so that no obvious trace can be left. The inscription is then repeated for each line till the whole designed number of periods is finished. Such inscription becomes possible with the high precision 2-D air bearing translation stages (Aerotech ABL1000).

Figure 1(b) and 1(c) are the images of the lines inscribed in this way for the 4th and 2nd order gratings, respectively. During the inscription, we set $v_{1}=90 \mu \mathrm{m} / \mathrm{s}, v_{2}=500 \mu \mathrm{m} / \mathrm{s}$ and the length of the line being $30 \mu \mathrm{m}$. The average energy of each laser pulses on the fiber is about $85 \mathrm{~nJ}$. As can be seen from Fig. 1(b), the width of these lines and their distances are uniform. The width of each line is measured less than $1 \mu \mathrm{m}$ which is narrow enough to allow for the inscription of the 2nd or higher order gratings for C- or L-band wavelengths. However, with narrow separation, laser pulses would be severely diffracted by existing lines when later lines are inscribed. Figure 1 (b) corresponds to a 4th order LBL FBG of 2000 periods with a period of $2.2 \mu \mathrm{m}$ and its transmission spectrum is shown in Fig. 2(a). Apparently, transmission attenuation of this 4 th order grating can reach up to $17 \mathrm{~dB}$ at the peak despite the grating is only $\sim 4 \mathrm{~mm}$.

Each inscribed line can be reasonably assumed to be a cylinder with a diameter of $\sim 700 \mathrm{~nm}$ as shown in Fig. 1(b), which was measured under a microscope (ZEISS). A stack of images for these lines were taken by moving the focus position of the microscope from one side of the fiber core to the other with an offset of $1 \mu \mathrm{m}$. Using the method described in reference [8], the phase contrast of the lines is constructed and shown in Fig. 2 (b), indicating a nominal average phase variation of 0.06 . The refractive index contrast of these lines to the unmodified area can be calculated by $\Delta n=\Delta \phi /(2 \pi) \cdot \lambda / d$, where $\Delta \phi$ is the phase contrast, $\lambda$ is the wavelength used for microscope illumination and $d$ is the diameter of the line. Thus, the index change can be found around $7 \times 10^{-3}$. It can be noticed from Fig.2(b) that the phase change is negative for the fs laser inscribed lines, implying a reduction of the refractive index in these positions. This is reasonable since the high intense laser repulses the material outwards leaving

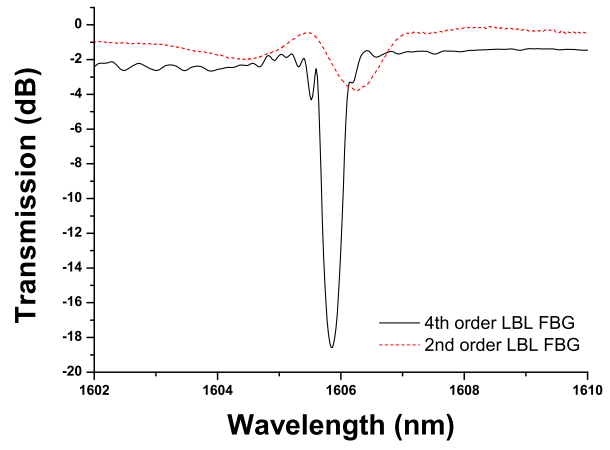

(a)

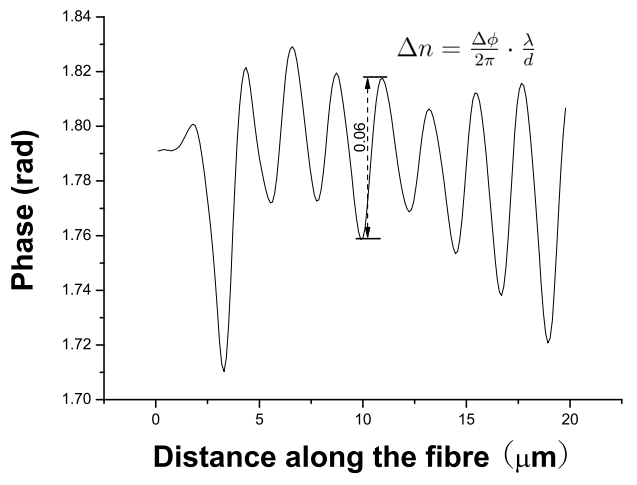

(b)

Figure 2. (a) Spectra of the LBL FBGs of 2nd and 4th orders. (b) The phase variation measured with the quantitative phase microscopy technique

a less dense region with reduced refractive index.

In comparison with the 1st order FBG reported in our previous work, which requires each point to be within the core, these lines go across the whole fiber core and thus relieve the demand of alignment significantly. For each line, the modification is the average result of a number of pulses instead of just one, and consequently, the change to the refractive index is more uniform, resulting in reduced phase noise and the insertion loss. For a grating of 2000 periods, the insertion loss is less than $0.5 \mathrm{~dB}$, which is obtained by finding the difference of the transmission at longer wavelength after the grating was written. The reason for the reduction to the loss compared to PBP FBG can be attributed to the larger size of the modified area, as it is lines that are disturbing the light waves in the core instead of point structures. It can be inferred that the insertion loss could be further reduced for an FBG inscribed by a plane-by-plane method.

Writing of the 2nd order LBL FBG is also attempted and Fig. 1(c) is the image of such a grating. Significant overlap between the neighboring lines can be noticed. Nevertheless, periodic structure can still be discerned, generating a weak grating response as shown in Fig.2(a).

The annealing of the 4th order LBL FBG at high temperature was carried out on another grating made in the same way but with the central wavelength at $1550 \mathrm{~nm}$. The grating was put into a furnace (carbolite, with the resolution $0.02^{\circ} \mathrm{C}$ ) with temperature set at $800^{\circ} \mathrm{C}$. We noticed that at $800^{\circ} \mathrm{C}$, the wavelength of the grating shifted $\sim 10 \mathrm{~nm}$ to longer wavelength 


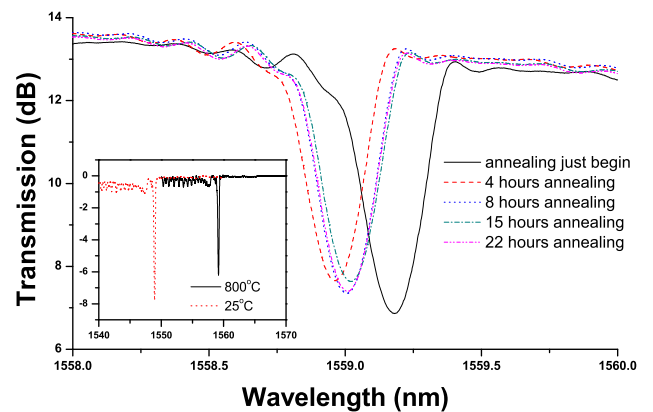

(a)

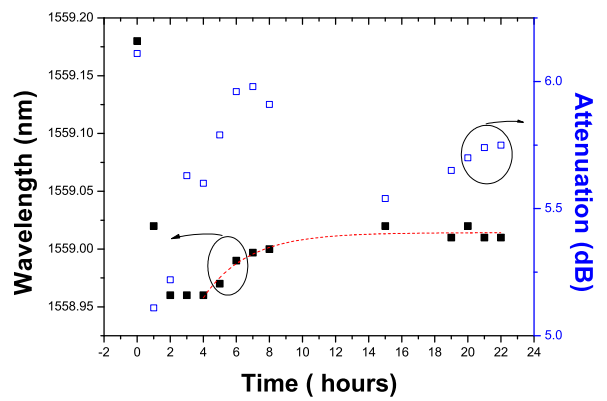

(b)

Figure 3. (a) Spectral evolution during the annealing process. (b) The change of the central wavelength and the strength of the peak attenuation during the annealing.

side, as shown in the inset of Fig. 3(a). Figure 3(a) shows the spectral evolution of the grating over 22 hours at annealing temperature $800^{\circ} \mathrm{C}$. No significant decrease of the strength of FBG attenuation can be noticed. Figure 3(b) shows the exact change of the central wavelength and the strength of the attenuation band during the annealing. As can be seen, in the first hour of the annealing, the strength of the grating decreases but then it is then recovered over the following 7 hours and then becomes more or less stable for the rest of the annealing time. Similarly, the Bragg wavelength presents a blue-shift for the first 2 hours and then it moves back and then gradually approaches a constant value, as shown by the trend line in Fig.3(b). The recovery of the wavelength and strength might be credited to the smoothing effect on the inscribed line structures during the annealing, which makes the grating more uniform.

The polarization dependent loss (PDL) of the grating (the one used for the annealing experiment) is also measured with the Luna OVA measurement system and the result is shown in Fig 4. In comparison, the PDL of the 1st order PBP FBG is also plotted in the figure. The length of the 1st order PBP FBG is $5 \mathrm{~mm}$ and the period is $0.545 \mu \mathrm{m}$ with the central wavelength at $1578 \mathrm{~nm}$. The transmission of both gratings are shown in the inset in Fig . 4, demonstrating similar attenuation. The PDL of the LBL FBG is only $0.5 \mathrm{~dB}$, whereas it reaches $\sim 7 \mathrm{~dB}$ for the PBP FBG. Nevertheless, the separation of the peaks coresponding to the two orthogonal polarization status in the PDL spectrum is almost the same for both grating, around $0.2 \mathrm{~nm}$. The much reduced PDL of the LBL grating can be mainly attributed to the fact that the inscribed periodic line structures are across whole fiber core, leading a much more symmetric index distribution and thus more even responce for

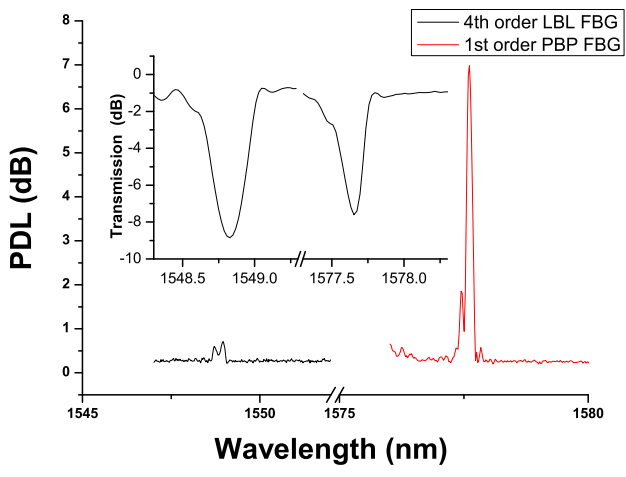

Figure 4. PDL spectrum of the 4th order LBL FBG and the 1st order PBP FBG. Inset: the transmission spectra of both gratings

the two polarisation states. The bandwidth of the 4th order grating is also broader than that of the 1st order grating since it has less number of period but almost the same length.

\section{CONCLUSIONS}

In conclusion, we describe the inscription of 4th order LBL FBGs using a fs laser with the aid of a high precision airbearing translation stage. The index modulation up to $7 \times 10^{-3}$ is achieved and high inband attenuation of $17 \mathrm{~dB}$ and small out-band insertion loss of less than $0.5 \mathrm{~dB}$ are obtained for a grating of 2000 periods. An annealing experiment at $800^{\circ} \mathrm{C}$ for 22 hours has been carried out and the results show such gratings can survive high temperature up to at least $800^{\circ} \mathrm{C}$ with little degradation. In comparison with PBP FBGs made with fs laser, the LBL FBGs possess much lower PDL.

\section{REFERENCES}

[1] K. Davis, K. Miura, N. Sugimoto, and K. Hirao, "Writing waveguides in glass with a femtosecond laser," OPTICS LETTERS, vol. 21, pp. 17291731, NOV 11996.

[2] Y. Cheng, K. Sugioka, K. Midorikawa, M. Masuda, K. Toyoda, M. Kawachi, and K. Shihoyama, "Control of the cross-sectional shape of a hollow microchannel embedded in photostructurable glass by use of a femtosecond laser," OPTICS LETTERS, vol. 28, pp. 55-57, JAN 1 2003.

[3] K. M. Zhou, Y. C. Lai, X. F. Chen, K. Sugden, L. Zhang, and I. Bennion, "A refractometer based on a micro-slot in a fiber bragg grating formed by chemically assisted femtosecond laser processing," Optics Express, vol. 15, pp. 15848-15853, 2007.

[4] S. Mihailov, C. Smelser, D. Grobnic, R. Walker, P. Lu, H. Ding, and J. Unruh, "Bragg gratings written in all-SiO2 and Ge-doped core fibers with 800-nm femtosecond radiation and a phase mask," JOURNAL OF LIGHTWAVE TECHNOLOGY, vol. 22, pp. 94-100, JAN 2004. Conference on Optical Fiber Communications Conference (OCF), ATLANTA, GEORGIA, 2003.

[5] A. Martinez, I. Khrushchev, and I. Bennion, "Direct inscription of Bragg gratings in coated fibers by an infrared femtosecond laser," OPTICS LETTERS, vol. 31, pp. 1603-1605, JUN 12006.

[6] Y. Lai, K. Zhou, K. Sugden, and I. Bennion, "Point-by-point inscription of first-order fiber bragg grating for c-band applications," Optics Express, vol. 15, no. 26, pp. 18318-18325, 2007.

[7] C. Hnatovsky, R. S. Taylor, E. Simova, V. R. Bhardwaj, D. M. Rayner, and P. B. Corkum, "Polarization-selective etching in femtosecond laserassisted microfluidic channel fabrication in fused silica," Opt. Lett., vol. 30, no. 14, pp. 1867-1869, 2005

[8] A. Roberts, E. Ampem-Lassen, A. Barty, K. Nugent, G. Baxter, N. Dragomir, and S. Huntington, "Refractive-index profiling of optical fibers with axial symmetry by use of quantitative phase microscopy," OPTICS LETTERS, vol. 27, pp. 2061-2063, DEC 12002. 